TITLE:

\title{
Amorphous diamond-like carbon film prepared by pulsed laser deposition with application of pulsed negative bias voltage
}

\section{AUTHOR(S):}

Matsuyama, Nobuyuki; Yukimura, Ken; Maruyama, Toshiro

\section{CITATION:}

Matsuyama, Nobuyuki ...[et al]. Amorphous diamond-like carbon film prepared by pulsed laser deposition with application of pulsed negative bias voltage. JOURNAL OF APPLIED PHYSICS 2001, 89(3): 1938-1941

\section{ISSUE DATE:}

2001-02-01

URL:

http://hdl.handle.net/2433/39697

\section{RIGHT:}

Copyright 2001 American Institute of Physics. This article may be downloaded for personal use only. Any other use requires prior permission of the author and the American Institute of Physics. 


\title{
Amorphous diamond-like carbon film prepared by pulsed laser deposition with application of pulsed negative bias voltage
}

\author{
Nobuyuki Matsuyama and Ken Yukimura \\ Department of Electrical Engineering, Faculty of Engineering, Doshisha University, Kyotanabe, \\ Kyoto 610-0321, Japan \\ Toshiro Maruyama ${ }^{\text {a) }}$ \\ Department of Chemical Engineering, Graduate School of Engineering, Kyoto University, Yoshida- \\ Honmachi, Sakyo-ku, Kyoto 606-8501, Japan
}

(Received 12 June 2000; accepted for publication 29 October 2000)

\begin{abstract}
Amorphous diamond-like carbon films were prepared by pulsed laser deposition with a synchronized application of pulsed negative bias voltage to the substrate. A beam from a $\mathrm{Nd}$ :yttrium-aluminum-garnet laser $(1064 \mathrm{~nm})$ was radiated to a graphite target at a laser fluence of $10 \mathrm{~J} / \mathrm{cm}^{2}$. The negative bias voltage was changed from 0 to $10 \mathrm{kV}$ at a repetition rate of $10 \mathrm{~Hz}$. The film was deposited on a Si (111) substrate at a chamber pressure of $5 \times 10^{-5}$ Torr for $120 \mathrm{~min}$. The effects of bias voltage on the structure of the film were discussed on the basis of the measured deposition rate, Raman spectra, refractive index, dynamic hardness, and surface roughness. The effect of self-sputtering on deposition rate was observed at a negative bias voltage above $0.6 \mathrm{kV}$. The application of bias voltage increased the fraction of $s p^{3}$ configuration in the film and made the film surface smoother. In particular, the application of $3 \mathrm{kV}$ gave the largest fraction of $s p^{3}$ bonding, and formed the hardest and smoothest film. Bias voltage above $5 \mathrm{kV}$, however, drastically reduced the hardness of the film. (C) 2001 American Institute of Physics. [DOI: 10.1063/1.1334640]
\end{abstract}

\section{INTRODUCTION}

Unhydrogenated amorphous diamond-like carbon (DLC) films have been prepared by pulsed laser deposition (PLD) $)^{1,2}$ at room temperature. The PLD method is different from that of traditional chemical vapor deposition where hydrogen plays an essential role by preferentially etching graphitic and amorphous carbon constituents. Some investigators ${ }^{3-8}$ have correlated the degree of diamond-like film character with deposition energy.

Kasi, Kang, and Rabalais, ${ }^{3}$ reported that the optimum $\mathrm{C}^{+}$energy range for formation of the diamond-like structure by ion-beam deposition is $30-175 \mathrm{eV}$. Below $10 \mathrm{eV}$ the final diamond-like structure has not been attained and above 180 $\mathrm{eV}$ there is a sharp increase in the dose required to attain this final structure. Lifshitz et al. ${ }^{4,5}$ proposed the subplantation (shallow implantation) model for film growth from hyperthermal $\left(\approx 1 \times 10^{3} \mathrm{eV}\right)$ species impinging on the substrate. The process included subsurface implantation, energy loss, preferential displacement of atoms with low displacement energies leaving high displacement energy atoms undisplaced, and sputtering of substrate material. The model, supported by ion-trajectory calculation and experimental data, was applied to diamond film formation from $\mathrm{C}^{+}$ions. Stevefelt and Collins ${ }^{6}$ reported a model of an expanding carbon plasma ablated from graphite feedstock by a focused laser beam. They showed that, under the experimental conditions of Marquardt, Williams, and Nage ${ }^{1}$ that have been proven relevant for producing hard DLC films characterized

\footnotetext{
a) Author to whom correspondence should be addressed: electronic mail: maruyama@cheme.kyoto-u.ac.jp
}

by a high percentage of $s p^{3}$ bonds, the plasma is composed of multiple-charged carbon ions with kinetic energies of the order of $1 \mathrm{keV}$. Cuomo et al. ${ }^{7,8}$ suggested that the condensation and growth of the DLC film was dependent on both the incident particle energy and a thermal quench at the substrate surface. They propose that thermally produced species of low energy will condense into the thermodynamically favored graphite structure when they reach the substrate. Alternatively, high-energy species may compact into the metastable diamond-like geometry when they impinge on the substrate. This geometry can be maintained if any excess energy that would drive it over the activation barrier toward the equilibrium structure is removed rapidly from the system.

There have been few reports about the effect of dc bias on the structure of the pulsed-laser-deposited DLC film. Sato et $a .^{2}$ reported that application of a negative dc bias $(-150$ to $-500 \mathrm{~V}$ ) during deposition by PLD using a $\mathrm{XeCl}$ laser was effective to ensure good quality of DLC film. Yamamoto et al. ${ }^{9}$ studied the effect of substrate dc bias $(-300$ to $200 \mathrm{~V}$ ) to the fraction of $s p^{3}$ bonding in the DLC film prepared by PLD using a KrF laser. Their results showed that the ion bombardment at above $200 \mathrm{eV}$ was effective to form $s p^{3}$ bonding.

This study describes the effect of a pulsed bias synchronized with the pulse of a Nd:yttrium-aluminum-garnet (YAG) laser on the preparation of DLC films. The effects of negative bias voltage on the structure of the film were discussed on the basis of measured scanning electron microscope (SEM), x-ray diffraction (XRD), Raman spectroscopy, ellipsometry, atomic force microscope (AFM), surface roughness, and dynamic hardness. 


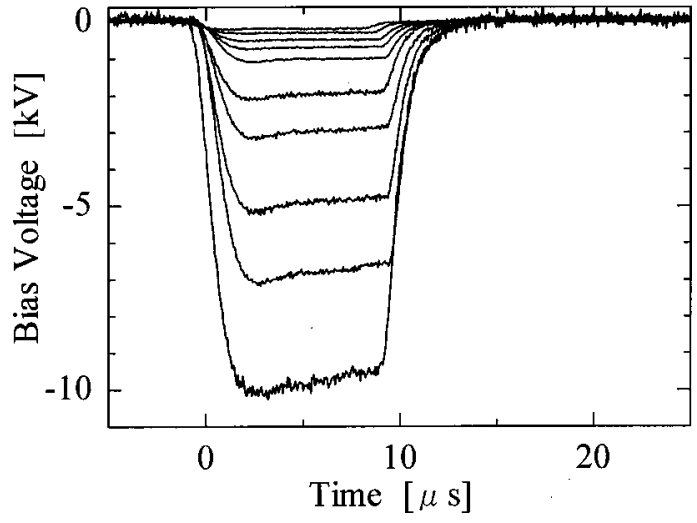

FIG. 1. Typical wave forms of pulsed bias voltage.

\section{EXPERIMENT}

A Nd:YAG laser (Spectron, SL-800) was operated at 10 Hz. Pulses of $1064 \mathrm{~nm}$ radiation were $25 \mathrm{~ns}$ in duration (temporal full width at half maximum) and had energy per pulse of $150 \mathrm{~mJ}$. The laser radiation was focused on the graphite target surface using a $30 \mathrm{~cm}$ focal length lens (Sigma Koki, SLSQ-50-300). The radiated target area, $0.0138 \mathrm{~cm}^{2}$, was calculated from the measured radiation area of the nonconcentrated laser on the basis of the fact that the spot size is proportional to the distance between the target surface and the lens focus. Thus, the laser fluence was $10 \mathrm{~J} / \mathrm{cm}^{2}$, and the laser power density was $4 \times 10^{8} \mathrm{~W} / \mathrm{cm}^{2}$. A $99.99 \%$ graphite rod (Nilaco, C-072591) was used as a target. The target was axially moved at a speed of $3 \mathrm{~mm} / \mathrm{min}$ with a motor to avoid any deep crater that may eject macroscopic graphite particle. A $10 \times 10 \mathrm{~mm}^{2}$ single crystal silicon was used as a substrate. The substrate was set in the position $60 \mathrm{~mm}$ from a target. The angle between a plane vertical to the substrate and the irradiation direction of plume was $35^{\circ}$. During the film deposition, the pressure in reaction chamber was kept at 5 $\times 10^{-5}$ Torr with a rotary pump and turbo-molecular pump. The deposition time was 120 min.

The reaction chamber and the target were grounded to make an anode, and the bias voltage was applied to the substrate holder. A pulse voltage of $10 \mu$ s in duration was used as the negative bias voltage. The $10 \mu$ s duration was selected to be long enough to cover the observed lifetime of an ablation plume. To apply the negative bias voltage to the plume, the $10 \mathrm{~Hz}$ voltage was triggered by the excitation lamp of the Nd:YAG laser. The trigger signal was amplified with a pulse generator (Hewlett Packard, 214B) and sent to a pulsed bias voltage source in time with the generation of the plume. Thus the rise of the pulsed bias was synchronized with that of the pulse of the laser. During the deposition, the wave form was monitored with an oscilloscope (Tektronix, TDS620A). Figure 1 shows typical wave forms of the pulsed bias voltage. The voltage change is approximately a rectangular form with a rise time of about $1.5 \mu$ s and duration of $10 \mu \mathrm{s}$.

For comparison, the DLC film was prepared by radiofrequency plasma deposition process with an application of pulsed negative bias voltage to the substrate. The radiofrequency power was $0.2 \mathrm{~kW}$. The applied pulse voltage was

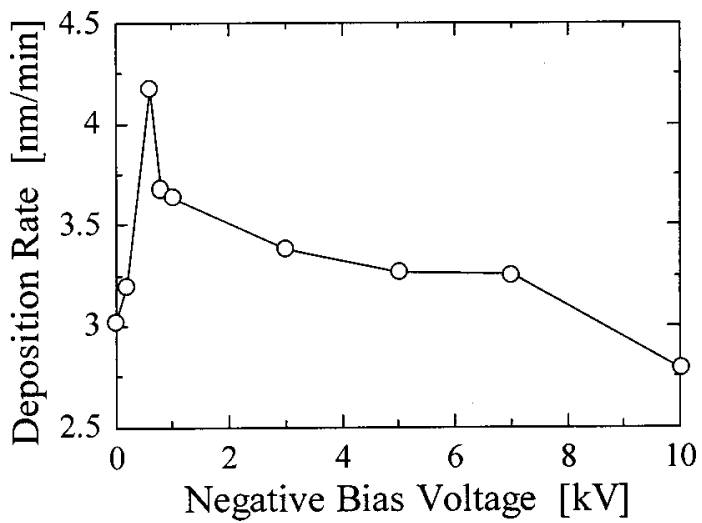

FIG. 2. Deposition rate of the DLC film as a function of negative bias voltage.

$-10 \mathrm{kV}$, the pulse width was $20 \mu \mathrm{s}$, and the frequency was $400 \mathrm{~Hz}$. The source gas was $\mathrm{CH}_{4}$, the flow rate of which was $27.9 \mathrm{sccm}$. A $10 \times 15 \mathrm{~mm}$ silicon substrate was placed on a 60-mm-diam copper target. The deposition was made at pressure of $10^{-3}$ Torr for $120 \mathrm{~min}$.

The morphology of the film was observed with SEM (Hitachi, SS2460N). The crystalline character of the film was checked by XRD (Rigaku, Mini Flex) using $\mathrm{Cu} K \alpha$ radiation. The Raman spectrum was measured with Raman spectrum meter (Renishaw, JRS-SYSTEM 2000) using the $514.5 \mathrm{~nm}$ line of an argon ion laser for excitation. The refractive index was measured with an ellipsometer (Mizojiri, DHA-XAVW/ S6). The surface roughness and film thickness were measured with a surface roughness meter using stylus instruments (Taylor-Hobson, Form Talysurf Series 2). In the measurement of surface roughness, a $3 \mathrm{~mm}$ scanning was made three times in an arbitrary direction. The film thickness was measured at the step that was made by masking a part of the substrate prior to the deposition. The surface roughness was also measured with an AFM (TopoMetrix, Explorer) in contact mode using a $\mathrm{Si}_{3} \mathrm{~N}_{4}$ cantilever. The average surface roughness was obtained by scanning the area of $100 \mu \mathrm{m}$ square. The hardness of the film was measured with a dynamic ultramicrohardness tester (Shimadzu, DUH-W201S) using a Berkovich indenter ( $115^{\circ}$ triangular pyramid) with a test load of $2 \mathrm{mN}$ at a loading speed of $0.711 \mathrm{mN} / \mathrm{s}$ for a duration of $5 \mathrm{~s}$.

\section{RESULTS AND DISCUSSION}

The XRD pattern showed that the prepared films were amorphous. The SEM observations confirmed that the surface of the film was very smooth and that there was not any prominent difference between the films with and without applying bias voltage. The smooth surface is characteristic of the DLC film.

Figure 2 shows the deposition rate of the film as a function of the negative bias voltage. Applying negative bias voltage in the range between 0.6 and $7 \mathrm{kV}$ enhances the deposition rate. However, the enhancement effect is not proportional to the negative bias voltage. It is largest at $0.6 \mathrm{kV}$ and decreases with increasing negative bias voltage. The ap- 


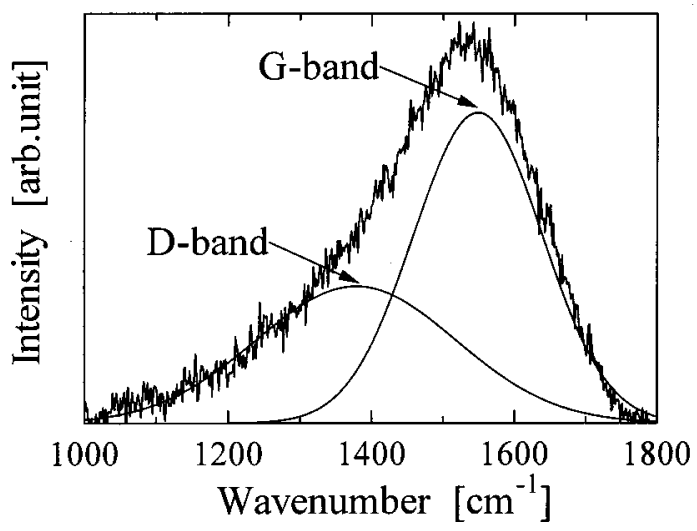

FIG. 3. Raman spectrum of PLD grown films without bias voltage.

plication of a negative bias voltage is inferred to be effective in enhancing the deposition rate by directing the plume ions to the substrate. However, a decrease in deposition rate can also occur because of a self-sputtering effect on the deposited film by the impinging ions. The results above $0.6 \mathrm{kV}$ show that the enhancement is largely overcome by the selfsputtering effect.

Figure 3 shows a typical example of the Raman spectrum for the film prepared without applying bias voltage. The spectrum shows only one broad asymmetric peak centered around $1530 \mathrm{~cm}^{-1}$, which is characteristic of DLC films. Thus, the unhydrogenated DLC film is obtained at laser power density of $4 \times 10^{8} \mathrm{~W} / \mathrm{cm}^{2}$. It is well below the reported critical level, $5 \times 10^{10} \mathrm{~W} / \mathrm{cm}^{2}$ at wavelength of 1060 $\mathrm{nm}^{1}$, and it is equal to the critical level, $\sim 10^{8} \mathrm{~W} / \mathrm{cm}^{2}$ at wavelength of $248 \mathrm{~nm}^{8}$, The signal can be decomposed into two peaks on the assumption that it consists of Gaussian peaks. Also shown in this figure are decomposed Gaussian peaks centered at 1390 and $1530 \mathrm{~cm}^{-1}$, which are called $D$ band and $G$ band, respectively. The $D$ band is associated with the stretching vibration of $s p^{2}$ clusters, and the $G$ band with the symmetric vibration mode-like contraction and expansion of the whole $s p^{2}$ cluster. The area ratio between the $D$ band and $G$ band, $S_{D} / S_{G}$, is used to express the fraction of ordered $s p^{2}$ clusters in the film.

Figure 4 shows the ratio $S_{D} / S_{G}$ as a function of negative bias voltage. The application of negative bias voltage de-

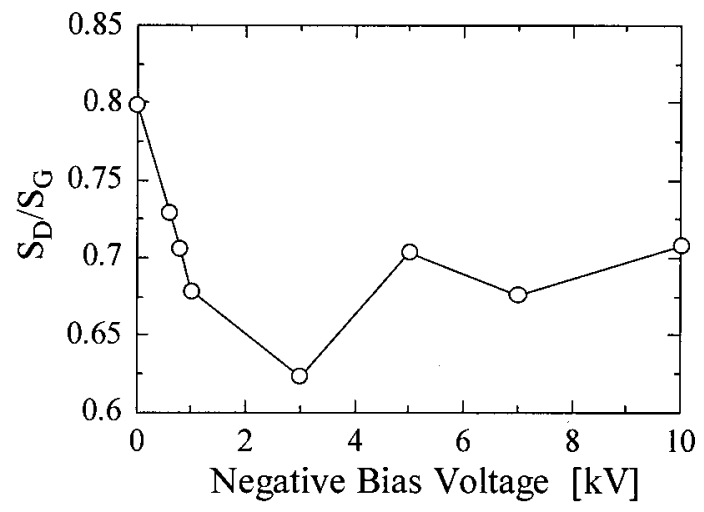

FIG. 4. Ratio $S_{D} / S_{G}$ as a function of negative bias voltage.

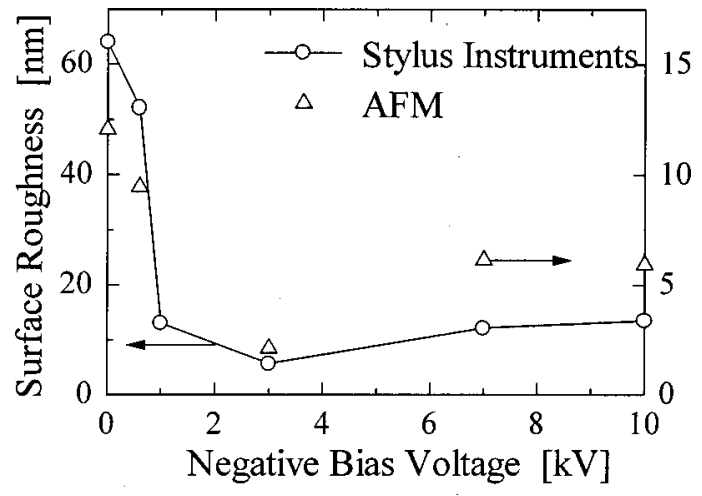

FIG. 5. Surface roughness of the DLC film measured by using stylus instruments and by using AFM.

creases the ratio from that obtained without applying negative bias voltage. In particular, the ratio shows the lowest value at $3 \mathrm{kV}$, where the effect of the self-sputtering on the deposition rate is apparent in Fig. 2. Thus the ion impact on the film is effective in decreasing the fraction of $s p^{2}$ bonds in the film on behalf of $s p^{3}$ bonds. The impact of an energetic ion may locally force surface $s p^{2}$ atoms into the metastable diamond-like configuration with preferential sputtering of the more weakly bonded carbon atoms in the growing film. At a negative bias voltage above $5 \mathrm{kV}$, however, excess energy may drive an atom back over the barrier to the equilibrium $s p^{2}$ structure, because that energy cannot be quickly removed. That is, the ion bombardment at $3 \mathrm{keV}$ is optimal for the reconstruction of the film. This result is consistent with the prediction of Stevenfelt and Collins ${ }^{6}$ that the plasma is composed of multiple-charged carbon ions with kinetic energies of the order of $1 \mathrm{keV}$ for producing hard DLC films characterized by a high percentage of $s p^{3}$ bonds.

Figure 5 shows the surface roughness of the film measured by using stylus instruments and by using AFM. The fact that the roughness of the former is about four times larger than that of the latter is attributable to the difference in mechanism for the measurements. The DLC film prepared by a radio-frequency plasma deposition process showed similar values of smoothness: $2.83 \mathrm{~nm}$ for AFM, and $15.5 \mathrm{~nm}$ for stylus instruments. The dependence on negative bias voltage fairly well agrees with each other, and the roughness of the film prepared at $3 \mathrm{kV}$ shows the smallest value. The dependence is also similar to that for the $S_{D} / S_{G}$ ratio, indicating that the surface of the film becomes smoother with increasing fraction of $s p^{3}$ bonds in the film. Self-sputtering by the impinging ions is also effective in making the smooth surface of the film.

Figure 6 shows the dynamic hardness and the refractive index of the film as a function of negative bias voltage. As expected, the hardness is very high and increases with increasing negative bias voltage to $3 \mathrm{kV}$, where the hardness shows a maximum value. The hardness of the DLC film prepared by radio-frequency plasma deposition process was 2000 , that is, one-fourth of the maximum value. Thus, the high value is attributable to the fact that the film prepared by PLD contains no hydrogen. In the meantime, the dynamic hardness shows a lower value at a negative bias voltage 


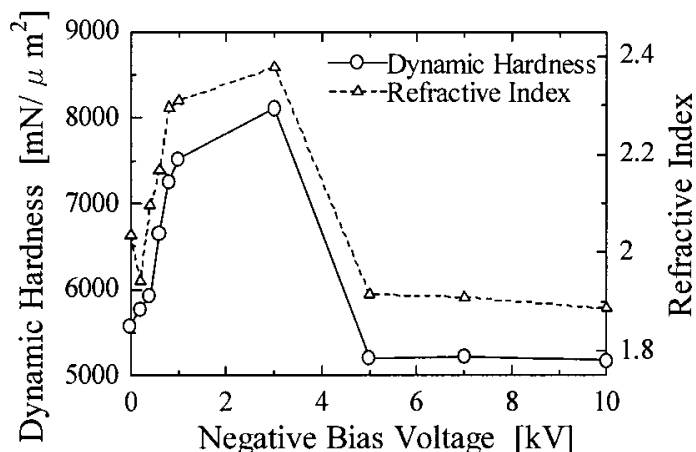

FIG. 6. Dynamic hardness and refractive index of the DLC film as a function of negative bias voltage.

above $5 \mathrm{kV}$. It is lower than that for the film prepared without applying negative bias voltage, being inconsistent with the dependences of the $S_{D} / S_{G}$ ratio and the surface roughness on negative bias voltage. This fact suggests that the ion bombardment with an excessive energy induces damage such as a decrease in scale of $s p^{3}$ cluster, in addition to the decrease in the fraction of $s p^{3}$ bond. The dependence of the refractive index on negative bias voltage is similar to that of the dynamic hardness. The refractive index (2.37) at $3 \mathrm{kV}$ is close to that (2.42) for the diamond, indicating that the hardest DLC film is obtained by PLD with applying negative bias voltage of $3 \mathrm{kV}$.

Thus, the ion bombardment breaks weak combinations at the surface of the film making the surface smooth. At the same time, the impact of energetic particles may locally force surface $s p^{2}$ atoms into the metastable diamond-like configuration, making the film hard with the highest refractive index. However, the ion bombardment with an excessive energy makes a structural change such as a decrease in scale of the $s p^{3}$ clusters.

\section{CONCLUSIONS}

Hard diamond-like carbon films can be prepared by pulsed laser deposition from a graphite target with a synchronized application of a pulsed negative bias voltage to the substrate. The effect of self-sputtering on deposition rate at a negative bias voltage above $0.6 \mathrm{kV}$ is observed. The application of bias voltage increases the fraction of $s p^{3}$ configuration in the film and makes the film surface smoother. In particular, the application of $3 \mathrm{kV}$ gives the largest fraction of $s p^{3}$ bonds, and forms the hardest and smoothest film. However, excessive negative bias voltage above $5 \mathrm{kV}$ drastically reduced the hardness of the film.

\section{ACKNOWLEDGMENTS}

The authors thank M. Sasaki of the Industrial Research Center of Shiga Prefecture for providing the facilities to measure the Raman spectrum, surface roughness, and refractive index, and N. Kanai of the Shimadzu Corporation for measuring the dynamic hardness of the film.

${ }^{1}$ C. L. Marquardt, R. T. Williams, and D. J. Nagel, Mater. Res. Soc. Symp. Proc. 38, 325 (1985).

${ }^{2}$ T. Sato, S. Furuno, S. Iguchi, and M. Hanabusa, Jpn. J. Appl. Phys., Part 2 26, L1487 (1987).

${ }^{3}$ S. R. Kasi, H. Kang, and J. W. Rabalais, J. Vac. Sci. Technol. A 6, 1788 (1988).

${ }^{4}$ Y. Lifshitz, S. R. Kasi, and J. W. Rabalais, Phys. Rev. Lett. 62, 1290 (1989).

${ }^{5}$ Y. Lifshitz, S. R. Kasi, J. W. Rabalais, and W. Eckstein, Phys. Rev. B 41, 10468 (1990).

${ }^{6}$ J. Stevefelt and C. B. Collins, J. Phys. D 24, 2149 (1991).

${ }^{7}$ J. J. Cuomo, D. L. Pappas, J. Bruley, J. P. Doyle, and K. L. Saenger, J. Appl. Phys. 70, 1706 (1991).

${ }^{8}$ D. L. Pappas, K. L. Saenger, J. Bruley, W. Krakow, J. J. Cuomo, T. Gu, and R. W. Collins, J. Appl. Phys. 71, 5675 (1992).

${ }^{9}$ K. Yamamoto, Y. Koga, S. Fujiwara, and F. Kokai, Jpn. J. Appl. Phys., Part 2 36, L1333 (1997). 